\title{
Discontinuing Infliximab in Benign Crohn's Disease: A Real-Life Population-Based Cohort Study
}

\author{
Lone Larsen $^{1}$, Asbjørn Mohr Drewes ${ }^{1,2}$, Jan Fallingborg ${ }^{1}$, Bent Ascanius Jacobsen ${ }^{1}$, Dalia Saleh Karzoun ${ }^{1}$, Hanna \\ Bjarkhamar Poulsen ${ }^{1}$ and Tine Jess ${ }^{2,3}$
}

${ }^{1}$ Department of Gastroenterology and Hepatology, Aalborg University Hospital, Denmark

${ }^{2}$ Department of Clinical Medicine, University of Aalborg, Denmark

${ }^{3}$ Department of Epidemiology Research, Statens Serum Institut, Denmark

Submission: May 05, 2020; Published: May 12, 2020

*Corresponding author: Lone Larsen, Department of Gastroenterology and Hepatology, Aalborg University Hospital, Mølleparkvej 4, DK-9000 Aalborg, Denmark

\begin{abstract}
Background/Aims: The need for maintenance therapy with infliximab after remission in patients with benign Crohn's disease remains to be studied. Our hypothesis was that patients with benign Crohn's disease did not need maintenance therapy after induction therapy if remission had been achieved.

Materials and methods: In a population-based regional Danish cohort of patients with Crohn's disease, we identified those initiating infliximab therapy from 2010 to $2016(n=312)$. As per protocol, those with predictors of severe prognosis (i.e. perianal -, fistulizing -, or extensive disease, young age, or previous surgery) continued on maintenance treatment as did those who had not obtained remission. We studied the benign cases with remission and no maintenance therapy.

Results: Of the 312 Crohn's disease patients exposed to infliximab induction therapy, 174 obtained remission, and of these 56 ( $31.5 \%$ ) were judged to be benign cases and were not scheduled for further infliximab treatment. Thirty of these patients (53.6\%) were still in remission after 6 months, 19 patients (33.9\%) after one year, and only 16 (28.6\%) after two years. Patients who relapsed within 6 months had higher CRP levels at initiation of infliximab therapy than individuals who did not relapse within 6 months (13.7 mg/L vs. $4.6 \mathrm{mg} / \mathrm{L} ; \mathrm{p}=0.002)$.

Conclusion: This study shows high relapse rates after infliximab induction therapy in benign Crohn's disease. Interestingly, Harvey Bradshaw Index and fecal calprotectin did not predict relapse, whereas high initial CRP levels did.

Keywords: Crohn's disease; Infliximab; Induction therapy; Maintenance therapy; Remission

Abbreviations: Anti-TNF: Anti-Tumor Necrosis Factor- $\alpha$; AZA: Azathioprine; CD: Crohn's Disease; IBD: Inflammatory Bowel Disease; IFX: Infliximab; IMM: Immunomodulators; HBI: Harvey Bradshaw Index; 6-MP: 6-Mercaptopurine; SCCAI: Short Clinical Colitis Activity Index; UC:
\end{abstract} Ulcerative colitis

\section{Introduction}

The introduction of anti-tumor necrosis factor- $\alpha$ (anti-TNF) has lead to improved remission rates and disease control in patients with Crohns disease [1]. The first available anti-TNF drug, infliximab, is still widely used to induce remission [2]. Although infliximab is relatively safe, it may increase risk of opportunistic infections [3], the risk of cancer [4] and treatment poses a burden to the health care system. Therefore, the potential for stratifying patients into those in need for long-term treatment and those who potentially could do with initial treatment needs further investigation.
The European Guidelines recommend maintenance therapy in all patients when remission is achieved by induction therapy [5]. The American Gastroenterological Association Guidelines also recommend maintenance therapy but focus on possible opportunistic infections such as tuberculosis or fungal infections as possible issues which can weigh towards termination of the therapy. Also in these guidelines, it is mentioned that long-term use of biologics are expensive [6]. According to the Danish national guidelines, treatment can be stopped if induction therapy induces remission [7]. Evidence behind the varying recommendations is 
limited, and real-life studies on remission rates after induction therapy in specific subgroups of patients from unselected population-based cohorts are lacking.

Randomized controlled studies, where patients are put on placebo after induction therapy have demonstrated that about $20 \%$ obtain long-term remission after mere induction therapy $[8,9]$. Furthermore several studies have demonstrated, that nearly all patients regain effect of the drug, when reintroduced in patients who relapse after a drug holyday [9]. The aim of the present study was to conduct a real-life population-based cohort study on the rate of relapse in patients with benign Crohn's disease after exposure to anti-TNF induction treatment only, and to assess the impact of potential predictors for relapse.

\section{Materials and Methods}

The study population was drawn from a population-based cohort of all patients with Crohn's disease $(n=\sim 1,200)$ from the North Denmark Region (583,000 inhabitants) [10]. All patients with Crohn's disease treated with infliximab during 2010-2016 were identified, using the database "GASTROBIO". Patients receiving biologic treatment for inflammatory bowel disease have been registered systematically in this web-based registry, which was established for both research purposes and for optimization of clinical management [11]. Patients with disease characteristics indicating a possible severe prognosis (i.e. perianal -, fistulizing -, or extensive disease, young age ( $<15$ years), or previous surgery, were all put on maintenance treatment and were excluded from this study. Patients with disease characteristics indicating a more benign prognosis, and who obtained remission after induction therapy with infliximab were considered for this study (Figure 1). Remission was evaluated clinically and defined as fecal calprotectin below $200 \mathrm{mg} / \mathrm{kg}$ and a HBI below 5 .

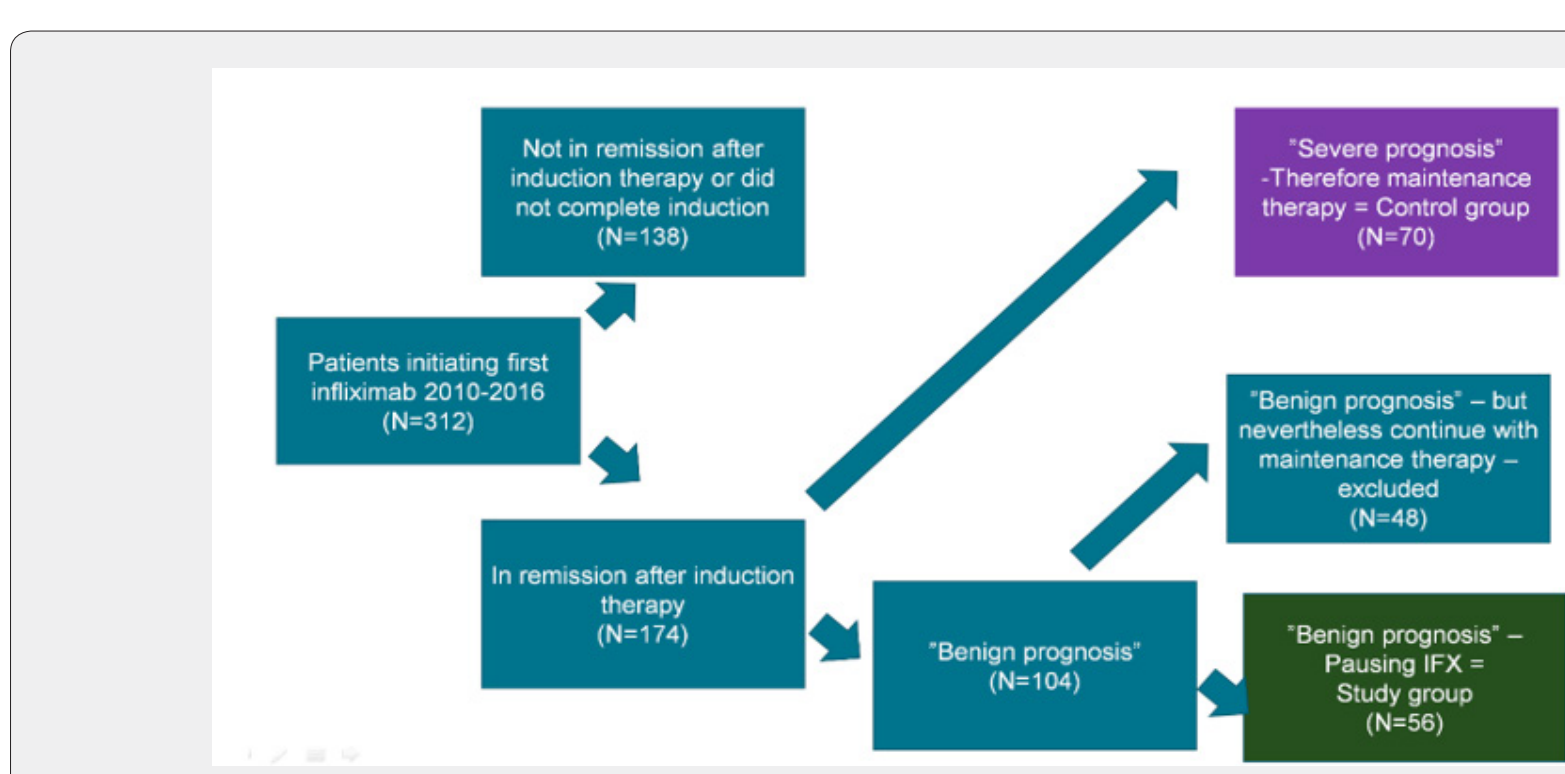

Figure 1: Flow-chart of infliximab treated patients from a population-based Crohn's disease cohort

\section{Time to relapse}

Time to relapse was measured as the difference between the dates of the third and fourth treatment. Early relapse was defined as retreatment within 6 months.

\section{Concomitant medication and markers of disease}

Use of concomitant treatment with azathioprine (AZA) or 6-mercaptopurine (6-MP) was registered and evaluated as one group, immunomodulators (IMM). Likewise, fecal calprotectin, C-reactive protein (CRP) and Harvey Bradshaw Index (HBI) was recorded in GASTROBIO at time of treatment initiation and at third infusion. CRP levels above $8 \mathrm{mg} / \mathrm{L}$, fecal calprotectin above $200 \mathrm{mg} / \mathrm{kg}$, and HBI above 5 were considered increased according to local laboratory standards.

\section{Statistical analyses}

Continuous variables were described using mean and standard deviation, and categorical variables were described with total numbers and percentages. Two-sided tests were applied with corresponding $\mathrm{p}$-values for all statistical analyses, using a significance level of $5 \%$. To assess differences between patients with or without retreatment regarding age at diagnosis, age at prescription, weight, and height, two-sided t-tests were performed. For the categorical variable "smoking", the difference between the early and late/no retreatment groups was assessed using chisquare test. Potential associations between disease markers (CRP, fecal calprotectin, and HBI) and disease relapse were tested with a t-test. CRP was further plotted as a dot plot of means. The log transformation was used to obtain normal distributed data and 
therefore the geometric means and coefficient of variation were reported.

Time to retreatment was illustrated by Kaplan-Meyer curves overall as well as according to CRP below/above 8 at treatment initiation, IMM/no IMM, using the log rank test for comparison. To further qualify time to retreatment analyses in the two CRP groups, as well as concomitant IMM treatment, Cox regression analysis was performed, and results were expressed as hazard ratios (HRs) with 95\% CIs. Analyses were performed using Stata (Stata/IC 12.1 for Windows, www.stata.com).

\section{Ethical considerations}

The study was approved by the Danish Board of Health (33013-720/1) and the Danish Data Protection Agency (2008-580028).

\section{Results}

From 2010 to 2016, 312 patients with Crohn's disease initiated infliximab therapy and of these, 70 who obtained remission, had disease characteristics indicating a possible severe prognosis (i.e. extensive disease, perianal location, previous surgery, fistulizing disease, young age $(<15)$ at debut) and were all put on maintenance treatment. One hundred and four patients who obtained remission had disease characteristics indicating a more benign prognosis (Figure 1). Of these 56 patients discontinued infliximab after induction ('induction only'). The remaining 48 patients continued with maintenance therapy based on an individual clinical assessment. Clinical characteristics are shown in Table 1. Thirty-two patients in the 'induction only' group (57.1\%) and 36 patients (51.4\%) in the maintenance group were females $(\mathrm{p}=0.59)$. The mean age at diagnosis was 26.8 years (range 15 to 52 years) in the 'induction only' group and 28.1 (range 8 to 63 years) in the maintenance group. Age at diagnosis and at prescription were comparable between the two groups $(\mathrm{p}=0.55$ and 0.34$)$. Twenty-two patients $(40 \%)$ in the 'induction only' group versus 17 patients $(25.4 \%)$ in the maintenance group were smokers $(\mathrm{p}=0.12)$.

Table 1: Characteristics of study cohort of infliximab treated patients with Crohn's disease receiving either induction therapy alone or maintenance therapy.

\begin{tabular}{|c|c|c|c|}
\hline & Induction Therapy & Maintenance Therapy & P-Value \\
\hline $\mathrm{N}$ & $56(100 \%)$ & $70(100 \%)$ & \\
\hline \multicolumn{4}{|l|}{ Gender } \\
\hline Female & $32(57.1 \%)$ & $36(51.4 \%)$ & $(p=0.59)$ \\
\hline Age at diagnosis, $\mathrm{N}$ & 56 & 70 & \\
\hline Mean (Std) & $26.8(9.7)$ & $28.1(13.7)$ & $(p=0.55)$ \\
\hline Age at prescription, $\mathbf{N}$ & 56 & 70 & \\
\hline Mean (Std) & $32.5(12.9)$ & $34.9(14.7)$ & $(p=0.34)$ \\
\hline Weight (kg), $\mathrm{N}$ & 43 & 52 & \\
\hline Mean (Std) & $71.2(16.2)$ & $72.6(22.5)$ & \\
\hline Height $(\mathrm{cm}), \mathrm{N}$ & 53 & 67 & \\
\hline Mean (Std) & $173.6(8.8)$ & $173.3(8.7)$ & \\
\hline Smoking habits at diagnosis, $\mathrm{N}$ & 55 & 67 & \\
\hline Yes & $22(40.0 \%)$ & $17(25.4)$ & $(p=0.12)$ \\
\hline Previous & $10(18.2 \%)$ & $14(20.9)$ & \\
\hline Never & $21(38.2 \%)$ & $35(52.2)$ & \\
\hline Unknown & $2(3.6 \%)$ & $1(1.5)$ & \\
\hline \multicolumn{4}{|c|}{ Immunomodulator at $3^{\text {rd }}$ dose infliximab } \\
\hline Yes & 31 & $\mathrm{~N} / \mathrm{A}$ & \\
\hline No & 25 & $\mathrm{~N} / \mathrm{A}$ & \\
\hline
\end{tabular}

\section{Time to retreatment}

Of the 56 patients with clinically judged benign Crohn's disease receiving induction therapy only, 30 (53.6\%) were still in remission after 6 months, whereas $28.6 \%$ had sustained remission after 2 years (Figure 2). Table 2 shows patients with need for early retreatment vs. patients with need for no or only later retreatment. There was no difference between these two groups regarding age at diagnosis (mean difference 1.8 years; $95 \% \mathrm{CI},-3.5$ to $7.0 ; \mathrm{p}=0.50$ ), age at infliximab prescription (mean difference 2.0 years; $95 \% \mathrm{CI},-4.9$ to $9.0 ; \mathrm{p}=0.55$ ), weight (mean difference 3.4 kilos; $95 \% \mathrm{CI},-6.8$ to $13.5 ; \mathrm{p}=0.50$ ), height (mean difference 3.5 centimeters; $95 \% \mathrm{CI},-1.3$ to $8.4 ; \mathrm{p}=0.15$ ), 


\section{Advanced Research in Gastroenterology \& Hepatology}

or smoking habits $(\mathrm{p}=0.91)$. Also, regression analyses revealed

or without concomitant immunomodulator treatment at the third no difference in time to retreatment between the patients with infliximab dose (HR 0.79; 95\% CI, 0.44 to $1.44 ; \mathrm{p}=0.48$ ).

Table 2: Characteristics of Crohn's disease patients in need or no need for early retreatment after infliximab induction therapy alone.

\begin{tabular}{|c|c|c|c|}
\hline N (\%) & Early Retreatment & Late/No Retreatment & P-Value \\
\hline & 26 (46.4\%) & $30(53.6 \%)$ & \\
\hline \multicolumn{4}{|l|}{ Gender } \\
\hline Female & $14(53.8 \%)$ & $18(60 \%)$ & \\
\hline Age at diagnosis, $\mathrm{N}$ & 26 & 30 & \\
\hline Mean (Std) & $27.8(10.5)$ & $26(9.1)$ & $(p=0.50)$ \\
\hline Age at prescription, $\mathrm{N}$ & 26 & 30 & \\
\hline Mean (Std) & $33.6(13.7)$ & $31.5(12.3)$ & $(p=0.55)$ \\
\hline Weight (kg), $\mathrm{N}$ & 19 & 24 & \\
\hline Mean (Std) & $73.1(17.1)$ & $69.7(15.7)$ & $(p=0.50)$ \\
\hline Height $(\mathrm{cm}), \mathrm{N}$ & 24 & 29 & \\
\hline Mean (Std) & $175.6(9.4)$ & $172.1(8.2)$ & $(p=0.15)$ \\
\hline Smoking habits at diagnosis, $\mathrm{N}$ & 29 & 26 & \\
\hline Yes & 10 & 12 & $(\mathrm{p}=0.50)$ \\
\hline Previous & 4 & 6 & \\
\hline Never & 10 & 11 & \\
\hline Unknown & 2 & 0 & \\
\hline \multicolumn{4}{|c|}{ Immunomodulator at $3^{\text {rd }}$ dose infliximab } \\
\hline Yes & 12 & 19 & $(p=0.20)$ \\
\hline No & 14 & 11 & \\
\hline
\end{tabular}

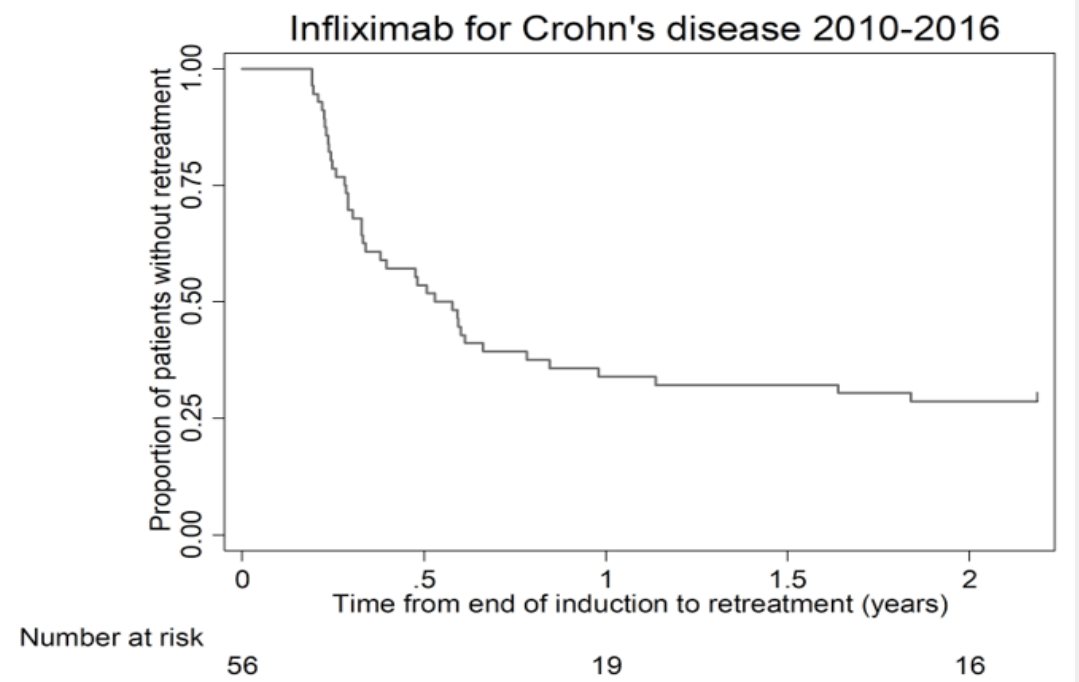

Figure 2: Time from planned termination of infliximab induction therapy to need for retreatment in a population-based Crohn's disease cohort.

\section{Predictors for sustained remission}

We assessed impact of HBI, fecal calprotectin, and CRP on prediction of sustained remission after induction therapy in patients with benign Crohn's disease. There was no difference in HBI at first $(\mathrm{p}=0.67)$ or third dose $(\mathrm{p}=0.94)$ between patients who relapsed (i.e. needed early retreatment) after induction therapy alone and patients who had sustained remission. Likewise, there 
was no difference in fecal calprotectin at first $(\mathrm{p}=0.86)$ or third dose $(p=0.78)$ between patients who did or did not relapse after induction therapy alone. However, a low CRP at initiation of therapy was a statistically significant predictor of sustained remission vs. (relapse $13.7 \mathrm{mg} / \mathrm{L}$ vs. no relapse: $4.6 \mathrm{mg} / \mathrm{L} ; \mathrm{p}=0.002$ ) (Figures 3 $\& 4)$. On the other hand, CRP at third dose was not a predictor of prognosis $(\mathrm{p}=0.53)$.

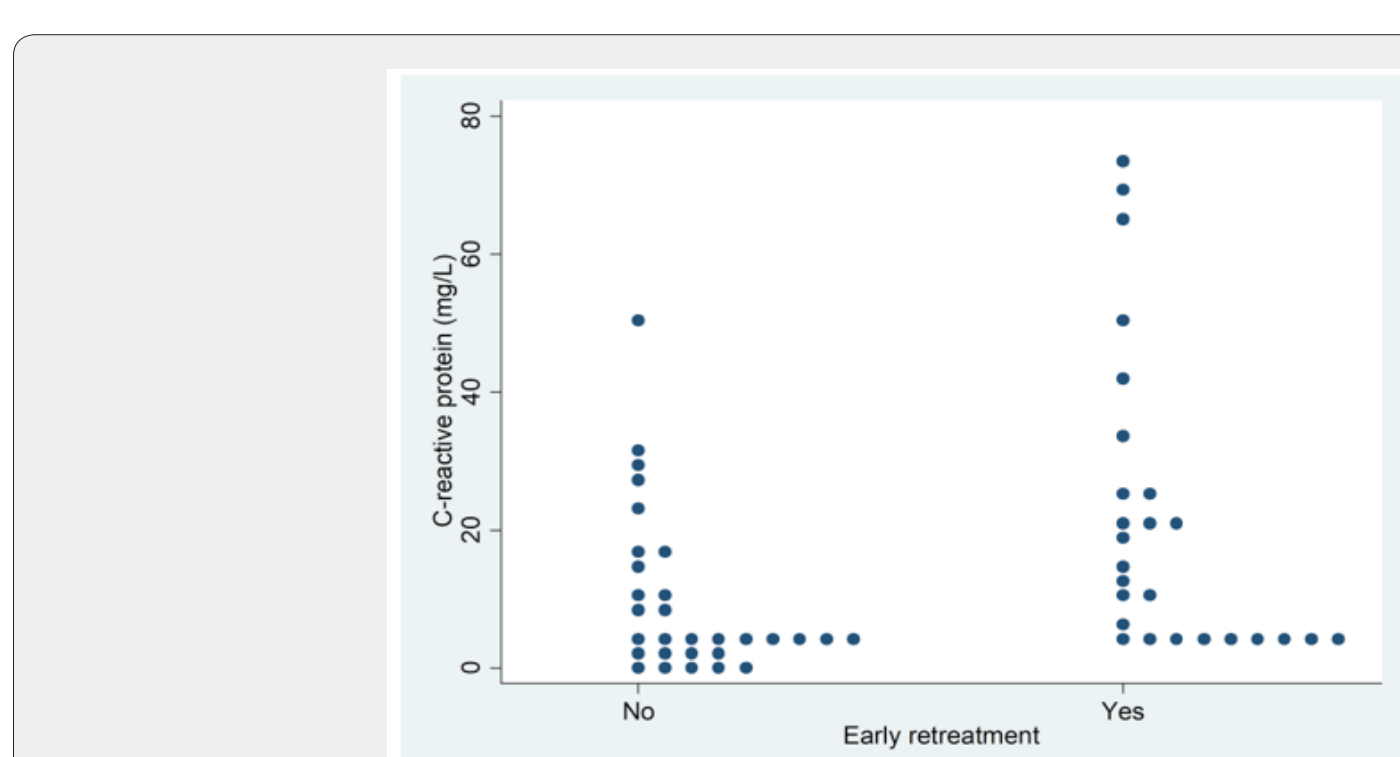

Figure 3: C-reactive protein (CRP) at initiation of infliximab induction therapy according to need for early retreatment in a population-based Crohn's disease cohort.

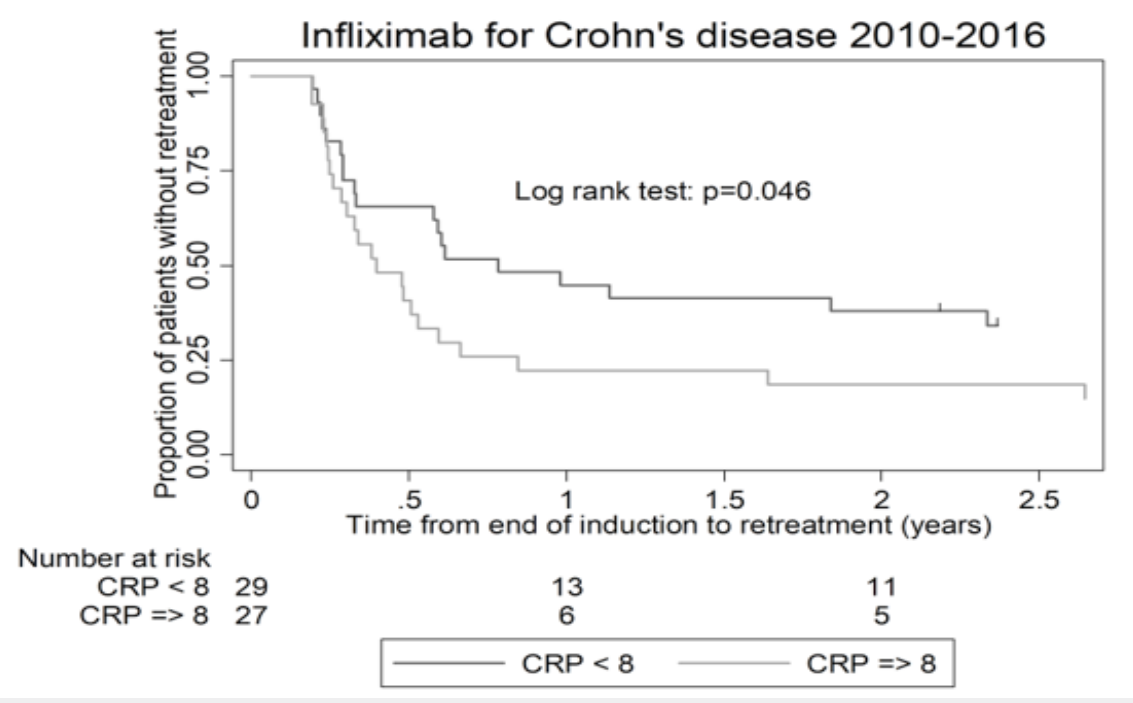

Figure 4: Proportion of patients needing retreatment with infliximab according to time since end of induction therapy in a population-based Crohn's disease cohort.

\section{Effect of re-treatment}

Of the 26 patients in the early retreatment group, 24 patients (92.3\%) regained response after re-treatment with IFX. One patient changed to another biological agent and one underwent surgery. No allergic reactions were reported.

\section{Discussion}

In the present real-life population-based cohort study of 312 patients receiving infliximab treatment in Northern Jutland during years 2010-2016, the effect of infliximab induction therapy alone in patients with benign Crohn's disease was evaluated. The present 
study demonstrated that among these patients, approximately $50 \%$ were in remission 6 months after end of induction therapy. One third were in remission after 1 year and just above one fourth after 2 years. Initial low CRP levels were predictive for sustained remission, whereas fecal calprotectin and HBI did not predict relapse.

\section{Strengths and limitations}

The primary strength of the present study was the real-life investigation of a geographically well-defined population covering a calendar period of 7 years. To our knowledge, this is the largest real-life study investigating remission after induction therapy alone in patients with benign Crohn's disease. Further, all patients in the North Jutland region of Denmark had been treated in one single center with the same core group of physicians managing patients throughout the entire period. The study also has potential limitations, which need to be considered. First, changes in prescription patterns and management of therapy could have occurred during the study period. Importantly, the study did not cover the first decade post-marketing hence avoiding early user bias. Second, the number of patients receiving induction therapy only was, despite the lack of previous similar data, of limited size, which may influence power in certain sub-analyses. Lastly, unmeasured confounding may occur.

\section{Comparison with previous studies}

Prior to the present study, a number of smaller and potentially more selected studies have reported varying results. In 2005, Domenech and colleagues compared induction therapy alone in 23 patients to scheduled maintenance in 27 patients. In patients with luminal Crohn's disease, who had complete response after induction therapy with or without maintenance with infliximab, two thirds maintained a sustained effect for more than two years [12]. However, they were almost all on concomitant immunomodulator therapy while this was the case for only half of our patients. Immunomodulator therapy has been shown to increase the effect of IFX which could explain this finding [13]. There was no significant difference in one year relapse rates between patients receiving induction therapy alone (relapse rate, $22 \%$ ) and patients receiving one year's maintenance therapy (relapse rate, 31\%). Likewise, in a comparable study with 92 patients, Chauvin and colleagues found no difference in relapse rates between the induction therapy only and maintenance group after one year (29 vs 44\%) [14]. A systematic review by Torres and colleagues including 32 prospective and retrospective cohort studies, but no unselected population-based studies concluded that approximately 55-60\% of patients who discontinued anti-TNF therapy would relapse within 2 years regardless of concomitant immunomodulator therapy [15].

\section{Predictors for response}

In the present real-life population-based unselected cohort study of 312 patients of whom 56 received induction therapy only, relapse rates were $46 \%$ after only 6 months and only $29 \%$ remained in remission after two years. Thus, even after careful clinical assessment of patients to determine whom would need induction therapy only, treatment appeared insufficient for half of the patients already within the first six months. It could be discussed whether other features, like for example smoking habits, should be part of our clinical assessment. Through examination of prospectively collected data on CRP, HBI, and fecal calprotectin, we assessed these factors as potential predictors of relapse and hence as potential factors for patient stratification. We found that even a slight elevation in CRP at initiation of therapy was a predictor for need for early retreatment. This is partly in accordance with previous studies reporting that elevated CRP levels are associated with an increased risk of short-term relapse [16]. It has previously been shown that an elevated CRP at discontinuation of therapy is associated with a higher risk of relapse [17]. As considered in this paper, some patients do not synthesize CRP despite active inflammation, thus potentially rendering false negative measurement. However, it is not surprising, that elevated CRP is a predictor for relapse, as these patients are likely to have active disease. Other studies looked at CRP after one year's treatment or even after induction therapy, and it has been shown that younger age at diagnosis, smoking and longer disease duration as well as high CRP and fecal calprotectin levels were factors associated with higher risk of relapse after discontinuation of infliximab maintenance therapy [18]. In contrast to findings for CRP, we did not find fecal calprotectin or HBI to differ between patients who relapsed and patients who remained in remission after induction therapy only.

Our finding of no impact of fecal calprotectin is quite surprising, since other studies have established an association between elevated fecal calprotectin levels and an increased risk of relapse [19]. However, we may have introduced selection bias by providing maintenance therapy to those with a fecal calprotectin level above $200 \mathrm{mg} / \mathrm{kg}$. Real life studies assessing HBI as a marker of relapse are lacking. Regarding exposure to immunomodulators, we did not observe an impact on risk of relapse. This is in contrast to a retrospective observational multicenter study from 78 Spanish centers suggesting that immunomodulator therapy at time of discontinuation of anti-TNF therapy was associated with a lower risk of relapse [20]. However, population-based studies for comparison are lacking.

\section{Conclusion}

In conclusion, our population-based real-life study of cessation of infliximab treatment after induction therapy in patients with benign Crohn's disease showed sustained remission after two years in one fourth of patients and this subgroup may represent a particular disease phenotype, where inflammation can be controlled with infliximab induction therapy exclusively. We identified CRP level at initiation of therapy as a potential patient stratifier, whereas fecal calprotectin, $\mathrm{HBI}$, and treatment with immunomodulators were not predictive of relapse. Our study underscores the need for development of algorithms that enable 
identification of patients in whom termination of treatment after induction therapy can be recommended.

\section{Acknowledgements}

We would like to thank Mikkel Kramme Abildtoft at Zitelab for assistance with output from the database.

\section{Funding}

This work was supported by the Heinrich Kopps Legat.

\section{References}

1. Targan SR, Hanauer SB, van Deventer SJ, Mayer L, Present DH, et al. (1997) A short-term study of chimeric monoclonal antibody cA2 to tumor necrosis factor alpha for Crohn's disease. Crohn's Disease cA2 Study Group. N Engl J Med 337(15): 1029-1035.

2. Van Assche G, Vermeire S, Rutgeerts $P$ (2009) Infliximab therapy for patients with inflammatory bowel disease: 10 years on. Eur J Pharmacol 623 Suppl: S17-25.

3. Lichtenstein GR, Feagan BG, Cohen RD, Salzberg BA, Diamond RH, et al. (2012) Serious infection and mortality in patients with Crohn's disease: more than 5 years of follow-up in the TREATTM registry. Am J Gastroenterol 107(9): 1409-1422.

4. Lichtenstein GR, Feagan BG, Cohen RD, Salzberg BA, Diamond RH, et al. (2014) Drug therapies and the risk of malignancy in Crohn's disease: results from the TREATTM Registry. Am J Gastroenterol 109(2): 212223

5. ECCO Statement 6F (CD 2016) ECCO Guidel. 2016.

6. Terdiman JP, Gruss CB, Heidelbaugh JJ, Sultan S, Falck-Ytter YT (2013) AGA Institute Clinical Practice and Quality Management Committee. American Gastroenterological Association Institute guideline on the use of thiopurines, methotrexate, and anti-TNF- $\alpha$ biologic drugs for the induction and maintenance of remission in inflammatory Crohn's disease. Gastroenterology 145(6): 1459-1463.

7. Jens Frederik Dahlerup, Jan Fallingborg, Christian Lodberg Hvas, Jens Kjeldsen, Lars Kristian Munck (2013) Biologisk terapi, behandling af IBD. Presented at the: Guideline: https://www.dsgh.dk/images/ Guidelines/pdf/bioterapiibd.pdf

8. Hanauer SB, Feagan BG, Lichtenstein GR, Mayer LF, Schreiber S, et al. (2002) Maintenance infliximab for Crohn's disease: the ACCENT I randomised trial. Lancet (London, England) 359(9317): 1541-1549.

9. Rutgeerts P, D'Haens G, Targan S, Vasiliauskas E, Hanauer SB, et al. (1999) Efficacy and safety of retreatment with anti-tumor necrosis factor antibody (infliximab) to maintain remission in Crohn's disease.

This work is licensed under Creative

Commons Attribution 4.0 License

DOI:10.19080/ARGH.2020.15.555905
Gastroenterology 117(4): 761-769.

10. Jacobsen BA, Fallingborg J, Rasmussen HH, Nielsen KR, Drewes AM, et al. (2006) Increase in incidence and prevalence of inflammatory bowel disease in northern Denmark: a population-based study, 1978-2002. Eur J Gastroenterol Hepatol 18(6): 601-606.

11. Larsen L, Drewes AM, Fallingborg J, Jacobsen BA, Jess T (2016) Touch screens as a tool in patient care in the IBD outpatient clinic. Scand J Gastroenterol 51(9): 1106-1110.

12. Domènech E, Hinojosa J, Nos $\mathrm{P}$, Garcia-Planella E, Cabré E, et al. (2005) Clinical evolution of luminal and perianal Crohn's disease after inducing remission with infliximab: How long should patients be treated? Aliment Pharmacol Ther 22(11-12): 1107-1113.

13. Colombel JF, Sandborn WJ, Reinisch W, Mantzaris GJ, Kornbluth A, et al. (2010) Infliximab, azathioprine, or combination therapy for Crohn's disease. N Engl J Med 362(15): 1383-1395.

14. Chauvin A, Le Thuaut A, Belhassan M, Le Baleur Y, Mesli F, et al. (2014) Infliximab as a bridge to remission maintained by antimetabolite therapy in Crohn's disease: A retrospective study. Dig Liver Dis 46(8): 695-700.

15. Torres J, Boyapati RK, Kennedy NA, Louis E, Colombel JF, et al. (2015) Systematic Review of Effects of Withdrawal of Immunomodulators or Biologic Agents From Patients With Inflammatory Bowel Disease. Gastroenterology 149(7): 1716-1730.

16. Gisbert JP, Marin AC, Chaparro M (2016) The Risk of Relapse after Anti-TNF Discontinuation in Inflammatory Bowel Disease: Systematic Review and Meta-Analysis. Am J Gastroenterol 111(5): 632-647.

17. Balzola F, Cullen G, Ho GT, Russell RK, Wehkamp J (2012) Maintenance of remission among patients with Crohn's disease on antimetabolite therapy after infliximab therapy is stopped. Inflamm Bowel Dis Monit 12(4): 152-153.

18. Gisbert JP, Marín AC, Chaparro M (2015) Systematic review: factors associated with relapse of inflammatory bowel disease after discontinuation of anti-TNF therapy. Aliment Pharmacol Ther 42(4): 391-405.

19. Brooks AJ, Sebastian S, Cross SS, Robinson K, Warren L, et al. (2017) Outcome of elective withdrawal of anti-tumour necrosis factor- $\alpha$ therapy in patients with Crohn's disease in established remission. J Crohns Colitis 11(12): 1456-1462.

20. Casanova MJ, Chaparro M, Garcia-Sanchez V, Nantes O, Leo E, et al. (2017) Evolution After Anti-TNF Discontinuation in Patients with Inflammatory Bowel Disease: A Multicenter Long-Term Follow-Up Study. Am J Gastroenterol 112(1): 120-131.

\section{Your next submission with JuniperPublishers} will reach you the below assets

- Quality Editorial service

- Swift Peer Review

- Reprints availability

- E-prints Service

- Manuscript Podcast for convenient understanding

- Global attainment for your research

- Manuscript accessibility in different formats

( Pdf, E-pub, Full Text, audio)

- Unceasing customer service

Track the below URL for one-step submission https://juniperpublishers.com/online-submission.php 